Rev. Inst. Flor. v. 27 n. 1 p. 103-115 jun. 2015

http://dx.doi.org/10.4322/rif.2015.008

ISSN impresso 0103-2674/on-line 2178-5031

\title{
QUANTIFICAÇÃO DA BIOMASSA EM PLANTIOS DE Pinus elliottii Engelm. em CLEVELÂNDIA - PR ${ }^{1}$
}

\section{MEASUREMENT OF BIOMASS IN PLANTATIONS OF Pinus elliottii Engelm.}

\author{
Gerson dos Santos LISBOA ${ }^{2,7}$; Karina LANZARIN³; David Fagner de Souza LIRA'; \\ Camila Mariah Magri PESCADOR ${ }^{4}$; Luciano Farinha WATZLAWICK ${ }^{5}$; Alvaro Felipe VALÉRIO ${ }^{6}$
}

RESUMO - Este trabalho teve como objetivo quantificar e modelar a biomassa em plantações de Pinus elliottii Engelm., com diferentes idades, no município de Clevelândia, Estado do Paraná. Os dados para a realização deste estudo foram provenientes de 25 povoamentos com idades de 1 a 25 anos, totalizando 125 árvores, sendo 5 para cada idade. As árvores foram derrubadas e seccionadas nos compartimentos: acículas, galhos vivos, galhos mortos, raízes, estruturas reprodutivas, madeira do fuste e casca do fuste. Em seguida, uma amostra de cada componente foi tomada para a obtenção de matéria seca. A ordenação da biomassa nos distintos componentes se distribuiu na ordem: madeira do fuste $>$ raiz $>$ casca $>$ galhos vivos $>$ acículas (estrutura fotossintética) $>$ galhos mortos e estruturas reprodutivas. Visando à obtenção de estimativas do peso de biomassa nos diferentes compartimentos da árvore por meio de variáveis dendrométricas, foram ajustados vários modelos matemáticos, entre eles, modelos tradicionalmente encontrados na literatura florestal. De uma maneira geral, a quantidade de biomassa da maioria dos componentes, apresentou alta relação com as variáveis dendrométricas, resultando em equações adequadas, exceto para os componentes galhos mortos e estruturas reprodutivas. As equações geradas para estimativa de peso total e dos componentes arbóreos da biomassa nas árvores de Pinus elliottii Engelm. são importantes ferramentas para análises técnicas, planejamento de projetos e estudos de viabilidade para uso da madeira.

Palavras-chave: floresta plantada; manejo florestal; ajuste de equações; modelagem da biomassa florestal.

\begin{abstract}
This study aimed to quantify and model the biomass in Pinus elliottii Engelm. plantations, with different ages, in the city of Clevelândia, state of Paraná. The data for this study came from 25 forest stands aged 1-25 years totaling 125 trees, 5 for each age. Trees were felled and sectioned in the compartments: needles, live branches, dead branches, roots, reproductive structures, wood stem and bark stem. Then, a sample was taken of each component for obtaining dry matter. Ordination of biomass in the different components are distributed in the order: wood stem $>$ root $>$ bark $>$ live branches $>$ needles (photosynthetic structure) $>$ dead branches and reproductive structure.
\end{abstract}

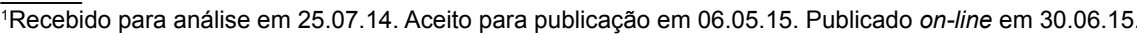

${ }^{2}$ Universidade Federal do Piauí - UFPI, BR 135, Km 3, Bairro Planalto Horizonte, 64900-000 Bom Jesus, PI, Brasil.

${ }^{3}$ Universidade Federal de Santa Maria - UFSM, Av. Roraima, 1000, 97105-900 Camobi, Santa Maria, RS, Brasil.

${ }^{4}$ E-mail - camilammp@yahoo.com.br

${ }^{5}$ Universidade Estadual do Centro-Oeste - UNICENTRO, R. Padre Honorino João Muraro, 875, Santa Cruz, 85015-430 Guarapuava, PR, Brasil.

${ }^{6}$ Prefeitura Municipal de Clevelândia, Praça Getúlio Vargas, n. 71, Centro, 85530-000 Clevelândia, PR, Brasil.

${ }^{7}$ Autor para correspondência: Gerson dos Santos Lisboa - gerson.lisboa@gmail.com
} 
Several mathematical models, among them models traditionally found in the forest literature, were adjusted in order to obtain estimates of biomass weight by dendrometric variables in different tree compartments. In general, the amount of biomass had good relations with the dendrometric variables resulting in satisfactory equations except for dead branches and reproductive structure components. The generated equations to estimate the total weight and arboreal components of the biomass in trees of Pinus elliottii Engelm. are important tools for technical analysis, project planning and feasibility studies for timber use.

Keywords: planted forest; forest management; adjustment of equations; modeling of forest biomass.

\section{INTRODUÇÃO}

Os grandes investimentos para o crescimento econômico dos países após a Segunda Guerra Mundial promoveu o desenvolvimento das atividades agrícolas, comerciais e industriais com base em práticas imediatistas e não sustentáveis, tais como, queimadas, conversão do uso original da terra e a consolidação do petróleo como principal matriz energética, promovendo a emissão de gases como Dióxido de Carbono $\left(\mathrm{CO}_{2}\right)$, Metano $\left(\mathrm{CH}_{4}\right)$, Óxido Nitroso $\left(\mathrm{N}_{2} \mathrm{O}\right)$, Clorofluorcarbonetos (CFCs), Hidrofluorcarbonetos (HFCs) e Hexafluoreto de Enxofre $\left(\mathrm{SF}_{6}\right)$ causadores do efeito estufa.

Algumas perspectivas apontam que o aumento da concentração do dióxido de carbono $\left(\mathrm{CO}_{2}\right)$ na atmosfera pode apresentar como consequência, a elevação da temperatura média global entre $1,5{ }^{\circ} \mathrm{C}$ a $4,5{ }^{\circ} \mathrm{C}$ até o ano de 2050 , o que ocasionaria transtornos de grandes proporções, procedentes da mudança do clima no planeta.

$\mathrm{O} \mathrm{CO}_{2}$ é o principal responsável pelo aquecimento global, sendo o gás mais emitido pelas atividades humanas e, devido a isto, existe um grande interesse em relação às florestas plantadas com rápido crescimento pela sua eficiência em fixação de carbono, havendo a necessidade do desenvolvimento de metodologias e técnicas adequadas para determinação e correto entendimento do volume de biomassa.

As florestas absorvem $\mathrm{CO}_{2}$ da atmosfera por meio da fotossíntese durante seu crescimento, armazenando grandes quantidades de carbono na biomassa de folhas, galhos, troncos e raízes, e liberando oxigênio de volta na atmosfera. As florestas tropicais da América, África e Ásia representam grandes reservatórios de carbono.
No entanto, elas estão sendo desmatadas em uma taxa de cerca de 8 milhões de hectares por ano. Na tentativa de manter as florestas tropicais e seus vastos estoques de carbono - intactas, a comunidade internacional está trabalhando para implementar políticas para compensar ações dos trópicos que reduzirem suas emissões de carbono provenientes de desmatamento e degradação florestal (Walker et al., 2011).

Estudos relacionados à quantificação de biomassa e carbono demandam trabalho, tempo e grande investimento financeiro. No entanto, sua determinação pode ser obtida por meio de métodos diretos e indiretos, nos quais o primeiro exige o corte das árvores e a pesagem de seus componentes separadamente e o segundo utiliza-se de dados precisos obtidos pelo método direto, para aplicação de técnicas de modelagem matemática ou de análise de imagens orbitais para a realização de estimativas.

A estimativa de biomassa é imprescindível aos estudos do balanço global de carbono (Ketterings et al., 2001). Assim, a avaliação acurada da biomassa florestal e seu padrão de mudança no tempo é um pré-requisito para ajudar a entender a função das florestas no ciclo do carbono (Sedjo, 1992; Fan et al., 1998; Brown et al., 1999).

Políticas bem-sucedidas irão exigir, entre outras coisas, o desenvolvimento de sistemas de medições florestais operacionais e de monitoramento para acompanhar os ganhos e perdas de carbono florestal ao longo do tempo. Este guia de campo descreve algumas ferramentas e técnicas básicas que provavelmente formariam a base de qualquer sistema de medição e monitoramento (Walker et al., 2011). 
Portanto, é de extrema importância o conhecimento da biomassa nos povoamentos florestais, bem como em seus distintos componentes. Para tanto, torna-se necessário o aperfeiçoamento de métodos de estimativas indiretas, buscando-se chegar a resultado confiável, por meio do desenvolvimento e uso de equações matemáticas apropriadas, utilizando dados originários de inventários florestais e assim, permitir o monitoramento e o entendimento das mudanças mais significativas nestas plantações, obtendo-se a quantidade de biomassa retida nas mesmas para uma determinada espécie. O presente estudo objetivou quantificar e modelar a biomassa em plantações de Pinus elliottii Engelm., com diferentes idades, no município de Clevelândia, Estado do Paraná.

\section{MATERIAL E MÉTODOS}

\section{1 Área de Estudo}

A área de estudo localiza-se no município de Clevelândia - PR, terceiro Planalto Paranaense (Figura 1). A vegetação natural é a Floresta Ombrófila Mista ou Floresta com Araucária (Instituto Brasileiro de Geografia e Estatística - IBGE, 2012). Conforme classificação de Köppen, o clima da região é caracterizado como Subtropical Úmido Mesotérmico ( $\mathrm{Cfb})$, sem estação seca definida e com ocorrência de intensas geadas no inverno. A temperatura média do mês mais quente é superior a $22{ }^{\circ} \mathrm{C}$ e do mês mais frio inferior a $18{ }^{\circ} \mathrm{C}$. A precipitação média anual varia de 1.980 a $2.100 \mathrm{~mm}$.

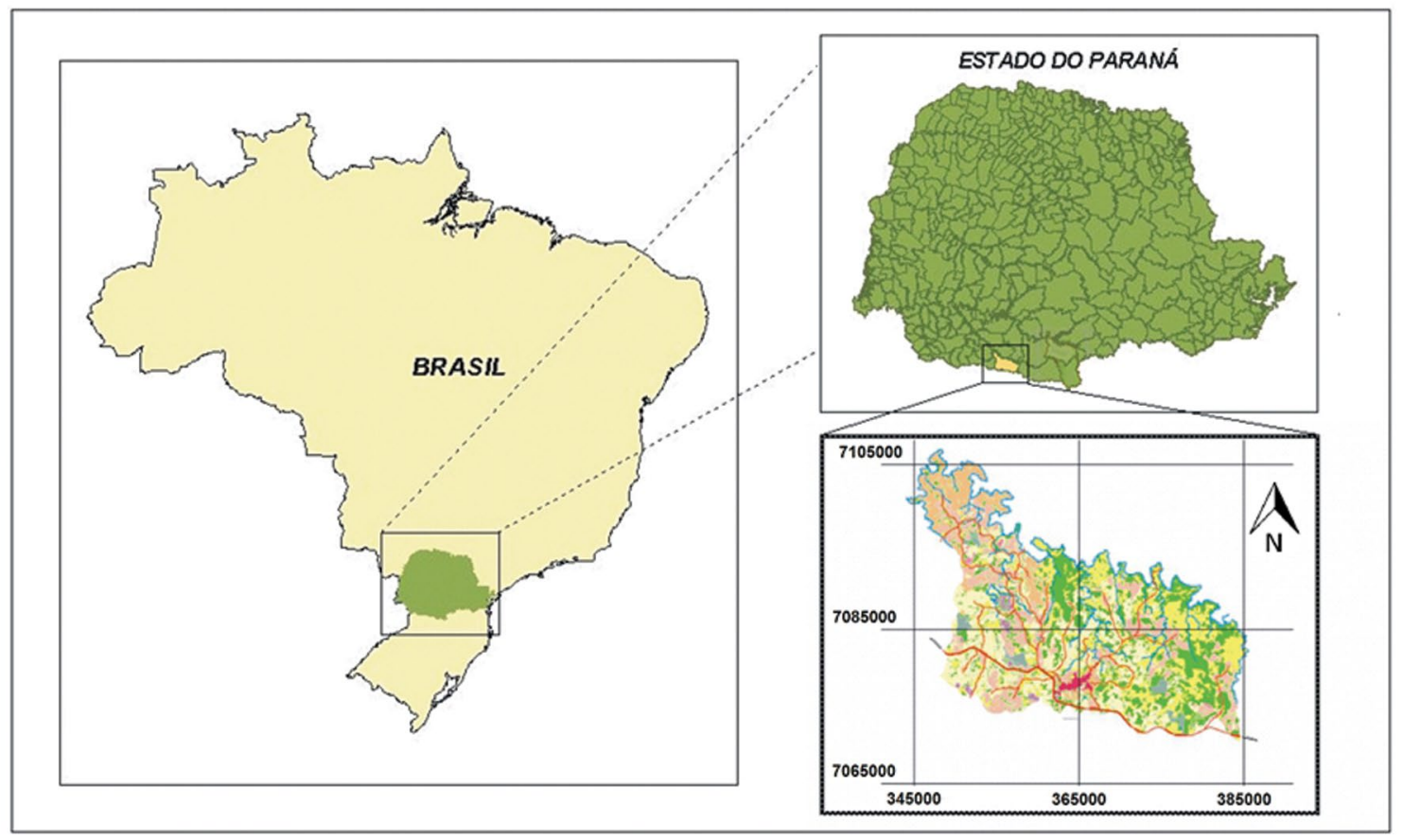

Figura 1. Croqui de localização da região de estudo, Clevelândia, PR.

Figure 1. Schematic illustration of study area location, Clevelândia, PR. 


\subsection{Amostragem e Quantificação da Biomassa}

Os levantamentos foram realizados em 25 povoamentos de Pinus elliottii Engelm., com idades de 1 a 25 anos, em propriedades pertencentes às empresas AFV Indústria e Comércio de Madeiras Ltda. e Camifra S/A Madeiras, Agricultura e Pecuária (Figura 2). O processo de amostragem utilizado foi o sistemático por meio do método de área fixa, sendo instaladas em cada povoamento unidades amostrais de $20 \mathrm{~m} \times 20 \mathrm{~m}\left(400 \mathrm{~m}^{2}\right)$.
Árvores com idade superior a dois anos tiveram a circunferência à altura do peito - 1,30 m - CAP medidas. Indivíduos com um ano de idade tiveram medidas a circunferência à altura do colo - CAC. Os valores de CAP foram convertidos em diâmetro à altura do peito - DAP. A semelhança dos tratos culturais e espaçamentos foi considerada na escolha das áreas selecionadas. Os inventários realizados para cada idade contemplaram amostragem superior a $1 \%$ da população, com um erro admissível de $10 \%$, com nível de probabilidade igual a $95 \%$.

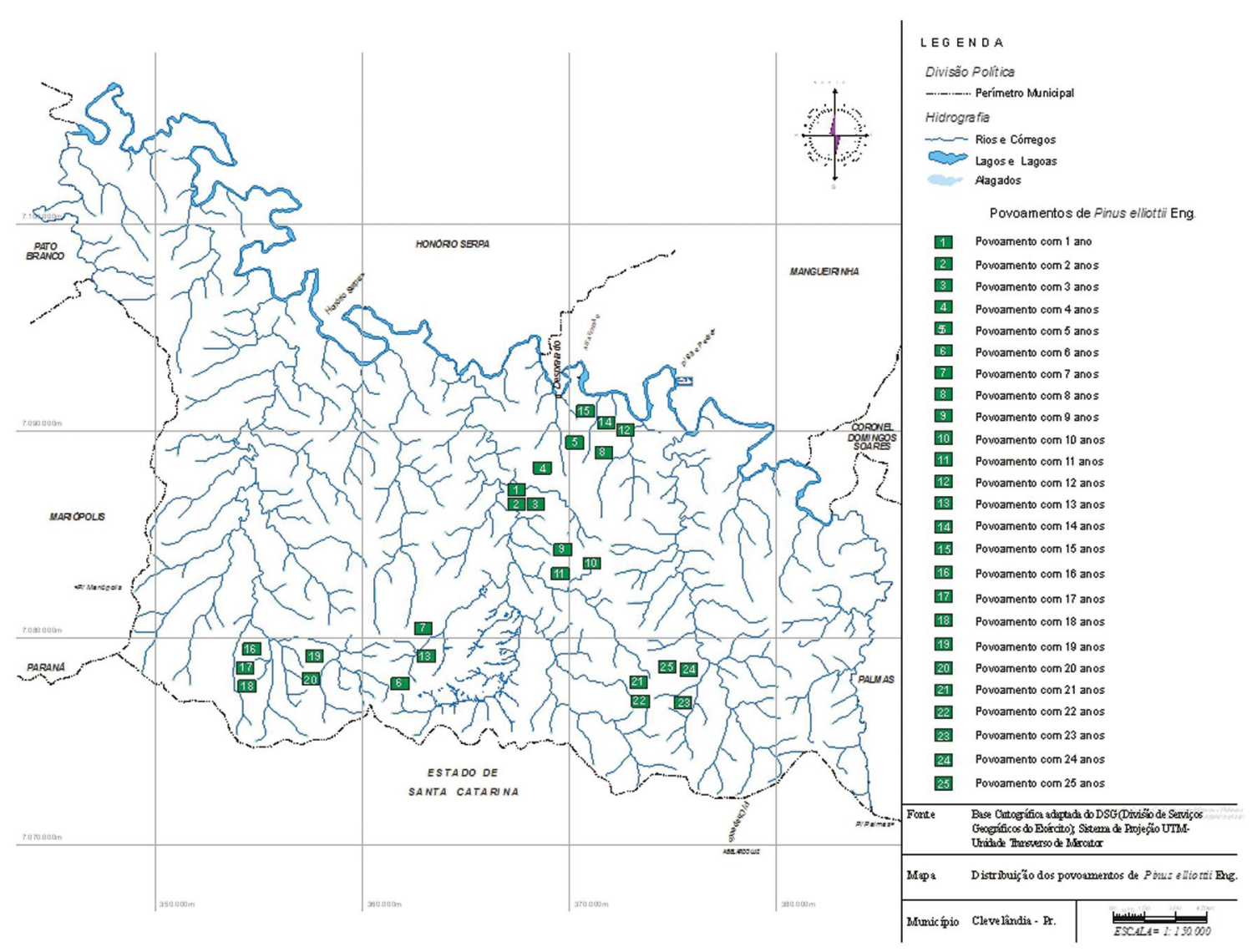

Figura 2. Localização dos povoamentos de Pinus elliottii Engelm., Clevelândia, PR.

Figure 2. Location of Pinus elliottii Engelm., Clevelândia, PR. 
Com base nos dados inventariados foram cortadas cinco árvores em cada idade, totalizando 125 indivíduos amostrados, os quais tiveram seus componentes arbóreos separados em: madeira do fuste, casca do fuste, acículas e galhos vivos, sendo pesados em campo para obtenção da massa de matéria fresca. Na determinação da biomassa das raízes, foram quantificadas as árvores de área basal média do povoamento $(\mathrm{dg})$ em cada idade, totalizando para este segmento amostra de raízes de 25 árvores. Os dados de biomassa foram coletados em campo no período de julho a dezembro de 2008, utilizando o método destrutivo. As amostras foram pesadas no campo em balança mecânica com precisão de $0,1 \mathrm{~g}$.

Para estimar o estoque de biomassa utilizaram-se como variáveis dependentes a quantidade total de biomassa de cada árvore e seus componentes, como variáveis independentes os valores advindos das medições de diâmetro à altura do peito - DAP, altura total (h) e a combinação de ambos.

Foram testados 17 modelos matemáticos para ajuste das equações de regressão, sendo 13 aritméticos e quatro logarítmicos, considerando cada um dos componentes da árvore, bem como a árvore inteira (Tabela 1).

Para selecionar a equação mais adequada para estimar a biomassa foram utilizadas as seguintes estatísticas: coeficiente de determinação ajustado ( $\mathrm{R}^{2}$ aj.), erro-padrão da estimativa corrigido em porcentagem (Syx\%), valor da estatística $\mathrm{F}$ e distribuição gráfica de resíduos em percentagem. Serão apresentados os resultados estatísticos e os gráficos de distribuição de resíduos que melhor se ajustaram para cada componente, assim como para a totalidade da árvore, com base nos critérios estatísticos acima citados.

Tabela 1. Modelos matemáticos testados para estimar a biomassa dos distintos componentes de Pinus elliottii Engelm., em Clevelândia, PR.

Table 1. Mathematical models tested to estimate the biomass of the different components of Pinus elliottii Engelm., in Clevelândia, PR.

\begin{tabular}{ll}
\hline N. & MODELO MATEMÁTICO \\
\hline 1 & $\mathrm{y}=b_{o} \mathrm{~d}^{b}$ \\
2 & $\mathrm{y}=b_{o}+b_{1} \mathrm{~d}+b_{2} d^{2}$ \\
3 & $\mathrm{y}=b_{o}+b_{1} \mathrm{~d}+b_{2}\left(\mathrm{~d}^{2} \mathrm{~h}\right)$ \\
4 & $\mathrm{y}=b_{o}+b_{1} \mathrm{~d}+b_{2} \mathrm{~d}^{2}+b_{3}\left(\mathrm{~d}^{2} \mathrm{~h}\right)$ \\
5 & $\mathrm{y}=b_{o}+b_{1} \mathrm{~d}^{2}+\mathrm{b}_{2}\left(\mathrm{~d}^{2} \mathrm{~h}\right)$ \\
6 & $\mathrm{y}=b_{o}+b_{1} \mathrm{~d}+\mathrm{b}_{2} \mathrm{~h}$ \\
7 & $\mathrm{y}=b_{o} \mathrm{~d}^{b} h^{c}$ \\
8 & $\mathrm{y}=b_{o}+b_{1} \mathrm{~d}$ \\
9 & $\mathrm{y}=b_{o}+b_{1} \mathrm{~d}^{2}$ \\
10 & $\mathrm{y}=b_{o}+b_{1} \mathrm{~d}^{3}$ \\
11 & $\mathrm{y}=b_{o}+b_{1} \mathrm{~d}^{2} \mathrm{~h}$ \\
12 & $\ln \mathrm{y}=b_{o}+b_{1} \ln \left(\mathrm{d}^{2} \mathrm{~h}\right)$ \\
13 & $\mathrm{y}=b_{o}+b_{1} \mathrm{~d}+b_{2} \mathrm{~d}^{2}+b_{3} \mathrm{~d}^{3}$ \\
14 & $\mathrm{y}=b_{o}+b_{1} \mathrm{~d}+b_{2} \mathrm{~d}^{2}+b_{3} \mathrm{~d}^{3}+b_{4} \mathrm{~d}^{4}$ \\
15 & $\mathrm{y}=b_{o}+b_{1} \mathrm{~d}+b_{2} \mathrm{~d}^{2}+b_{3} \mathrm{~d}^{3}+b_{4} \mathrm{~d}^{4}+b_{5} \mathrm{~d}^{5}$ \\
16 & $\ln \mathrm{y}=b_{o}+b_{1} \ln \mathrm{h}$ \\
17 & $\mathrm{y}=b_{o}+b_{1} \mathrm{~d}^{2}+b_{2} \mathrm{~h}^{2}+b_{3}\left(\mathrm{~d}^{2} \mathrm{~h}\right)$ \\
\hline $\mathrm{y}=$ peso de biomassa $; b_{o}, b_{1}, b_{2}, \ldots=\operatorname{coeficientes} ; \mathrm{d}=$ diâmetro à altura do peito; $_{\mathrm{h}=\text { altura total da árvore. }}$ \\
\hline
\end{tabular}




\section{RESULTADOS E DISCUSSÃO}

Na Tabela 2 são apresentados modelos da equação para estimar a biomassa total (parte aérea e raízes) em povoamentos de Pinus elliottii Engelm., com base na análise estatística e distribuição gráfica dos resíduos. Destaca-se como a mais apropriada para estimativa de biomassa total a equação 1, apresenta sua maior simplicidade, demandando apenas uma variável independente, como também, que a variável altura total (h) não expressa aumento da eficácia no ajuste dos modelos analisados, visto as condições de diferentes idades dos indivíduos (1 a 25 anos).

A distribuição dos resíduos da equação de melhor desempenho (Equação 1) pode ser observada na Figura 3, na qual se verifica que explicam de maneira satisfatória as variáveis de interesse, não obtendo uma distribuição de resíduos tendenciosa.

Tabela 2. Resultado das equações com melhor estatística para estimativa da totalidade de biomassa em povoamentos de Pinus elliottii Engelm., Clevelândia, PR.

Table 2. Result of equations with the best statistics to estimate the total biomass in Pinus elliottii Engelm., Clevelândia, PR.

\begin{tabular}{|c|c|c|c|c|c|}
\hline & $\mathrm{N}$. & EQUAÇÃO & $\mathrm{R}^{2} \mathrm{aj}$. & Syx $\%$ & $\mathrm{~F}$ \\
\hline \multirow{4}{*}{ 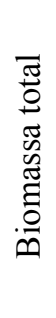 } & 1 & $\mathrm{y}=0,18838 \mathrm{~d}^{2,17493}$ & 0,9729 & 29,0732 & 862,657 \\
\hline & 10 & $y=27,63326+0,01010 d^{3}$ & 0,9497 & 20,4132 & 435,4573 \\
\hline & 12 & $\mathrm{y}=0,27834(\mathrm{dh})^{0,730605}$ & 0,9731 & 30,2700 & 832,9390 \\
\hline & 15 & $\begin{array}{l}y=156,12867-70,35593 d+9,35952 d^{2} \\
-0,48389 d^{3}+0,01132 d^{4}-0,0000942 d^{5}\end{array}$ & 0,9476 & 18,8565 & 84,1265 \\
\hline
\end{tabular}

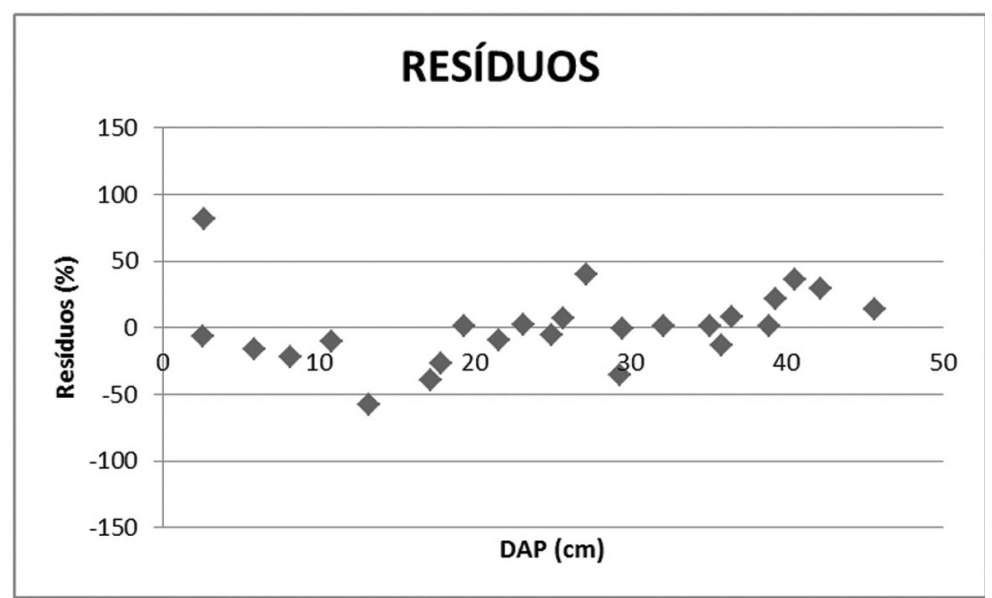

Figura 3. Distribuição dos resíduos para a equação número 1 na estimativa de biomassa total de Pinus elliottii Engelm.

Figure 3. Distribution of waste to the equation number 1 in the estimated total biomass of Pinus elliottii Engelm. 


\subsection{Ajuste de Equações para Peso de Biomassa dos Componentes Acima do Solo}

Os resultados dos ajustes da equação de melhor desempenho que exprimem a biomassa aérea, compreendido como aquele alocado nos componentes da árvore acima do solo, são apontados na Tabela 3.

A equação 1 apresentou coeficientes de determinação adequados e estatística $\mathrm{F}$ na estimativade biomassa aérea, sendo eles 0,9726 e 4405,556, respectivamente. Outras equações (7 e 12) tiveram desempenhos semelhantes para as estatísticas avaliadas, porém a escolha da equação 1 se tornou mais apropriada do ponto de vista prático, sendo necessária somente uma variável independente de fácil obtenção.

A distribuição dos resíduos pode ser observada na Figura 4, na qual se verificou que a equação logarítmica 1 explicou de maneira satisfatória a variável de interesse, isenta de tendenciosidade.

Tabela 3. Resultado da equação com melhor estatística para estimativa de biomassa aérea em povoamentos de Pinus elliottii Engelm., Clevelândia, PR.

Table 3. Result of equation with the best statistics to estimate above ground biomass in Pinus elliottii Engelm., Clevelândia, PR.

\begin{tabular}{llllll}
\hline & N. & EQUAÇÃO & R 2 aj. & Syx\% & F \\
\hline Biomassa aérea & 1 & $\mathrm{y}=0,14504 \mathrm{~d}^{2,21232}$ & 0,9726 & 29,8498 & 4405,5456 \\
\hline
\end{tabular}

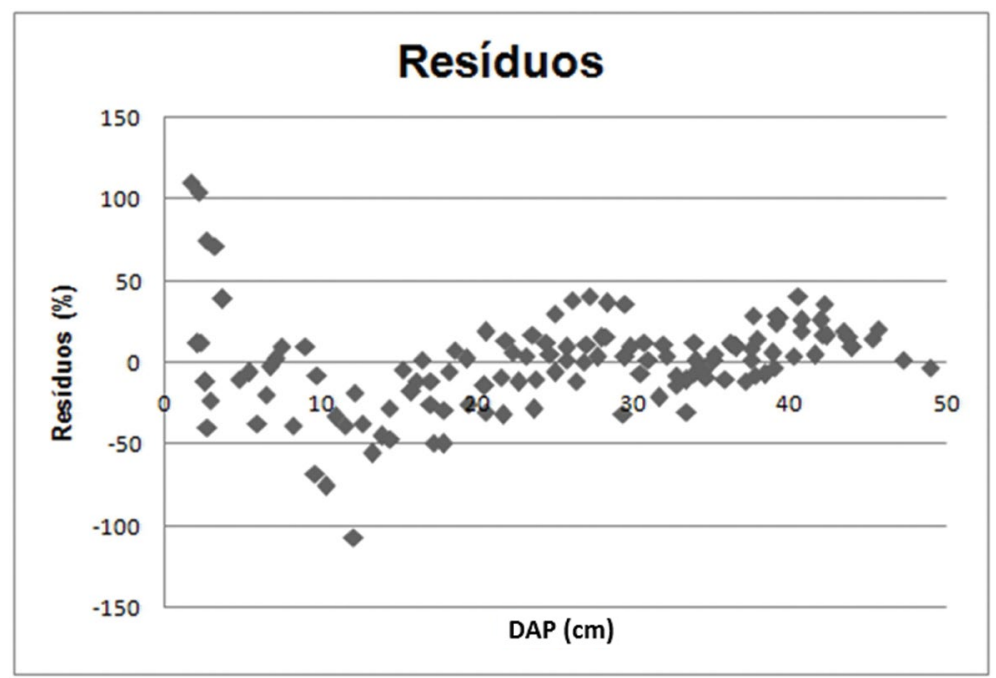

Figura 4. Distribuição dos resíduos para a equação número 1 na estimativa da biomassa acima do solo de Pinus elliottii Engelm.

Figure 4. Distribution of waste to the equation number 1 in the estimated above ground biomass of Pinus elliottii Engelm. 


\subsection{Ajuste de Equações para Estimativa de Biomassa da Madeira do Fuste}

A equação número 1 apresentou melhores estatísticas para estimativas de biomassa da madeira do fuste, descrevendo estatística com $\mathrm{R}^{2}$ aj. de 0,9718 e Syx $\%$ de $34,84 \%$ referentes à estimativa de biomassa. Na Tabela 4, pode ser visualizado o modelo da equação. Outras equações (7 e 12) obtiveram desempenhos semelhantes para as estatísticas avaliadas. Destacou-se a equação 1 como mais apropriada devido à praticidade e facilidade da obtenção de apenas uma variável independente. O gráfico de resíduos (Figura 5), referente à equação ajustada para biomassa da madeira do fuste, mostra que a equação logarítmica apresentou boa distribuição residual, sem tendência de super ou subestimativa ao longo da linha de regressão.

Tabela 4. Resultado da equação com melhor estatística para estimativa de biomassa da madeira do fuste em povoamentos de Pinus elliottii Engelm., Clevelândia, PR.

Table 4. Result of equation with the best statistics to estimate stem wood biomass in Pinus elliottii Engelm., Clevelândia, PR.

\begin{tabular}{llllll}
\hline & N. & EQUAÇÃO & R 2 aj. & Syx\% & F \\
\hline Biomassa do fuste & 1 & $\mathrm{y}=0,03389 \mathrm{~d}^{2,54292}$ & 0,9718 & 34,843 & 4271,857 \\
\hline
\end{tabular}

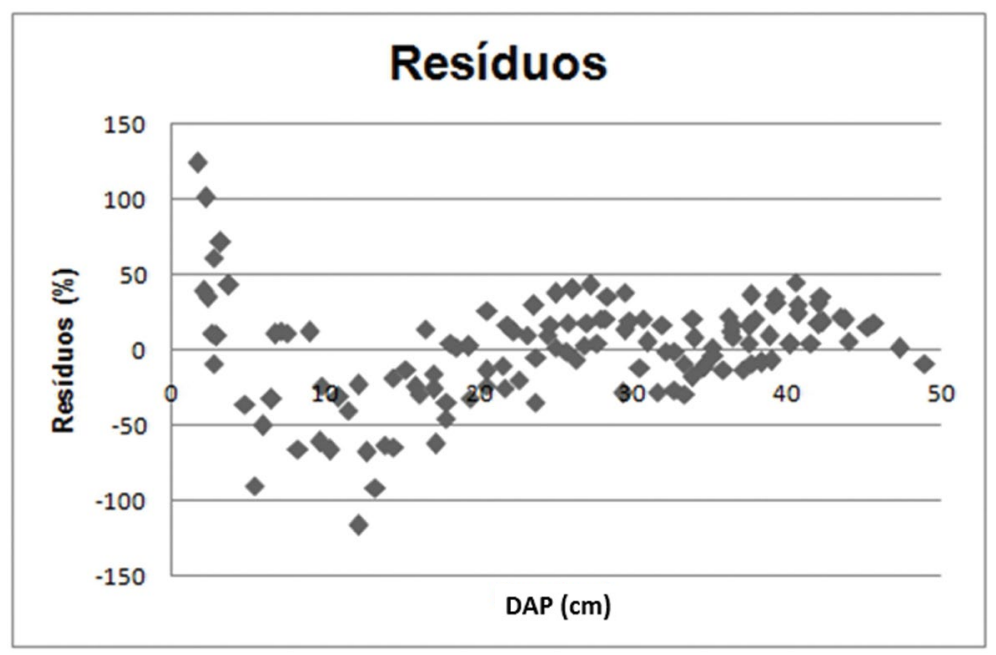

Figura 5. Distribuição dos resíduos para a equação número 1 na estimativa da biomassa da madeira do fuste de Pinus elliottii Engelm.

Figure 5. Distribution of waste to the equation number 1 in the estimated stem wood biomass of Pinus elliottii Engelm. 


\subsection{Ajuste de Equações para Estimativa de Biomassa das Acículas}

Os resultados dos ajustes dos modelos de equações que exprimem a biomassa das acículas de P. elliottii, entendida como a estrutura fotossinteticamente ativa, em kg, estão apontados na Tabela 5 .

A equação selecionada para estimativa de biomassa das acículas apresenta $\mathrm{R}^{2} \mathrm{aj}$. de 0,9084 e Syx\% de 34,4024\%, cuja distribuição dos resíduos pode ser observada na Figura 6. As equações resultantes dos modelos logarítmicos 1 e 12 apresentaram melhor distribuição gráfica de resíduos para a biomassa das acículas. É importante salientar que o ajuste resultante do modelo 1 se destaca do ponto de vista prático, pois demanda apenas uma variável independente de fácil obtenção.

Tabela 5. Resultado da equação com melhor estatística para estimativa de biomassa das acículas em povoamentos de Pinus elliottii Engelm., Clevelândia, PR.

Table 5. Result of the equation with the best statistics to estimate needles biomass in Pinus elliottii Engelm, Clevelândia, PR.

\begin{tabular}{llllll}
\hline & N. & EQUAÇÃO & R$^{2}$ aj. & Syx\% & F \\
\hline Biomassa das acículas & 1 & $\mathrm{y}=0,16513 \mathrm{~d}^{1,34367}$ & 0,9084 & 34,3024 & 1230,62 \\
\hline
\end{tabular}

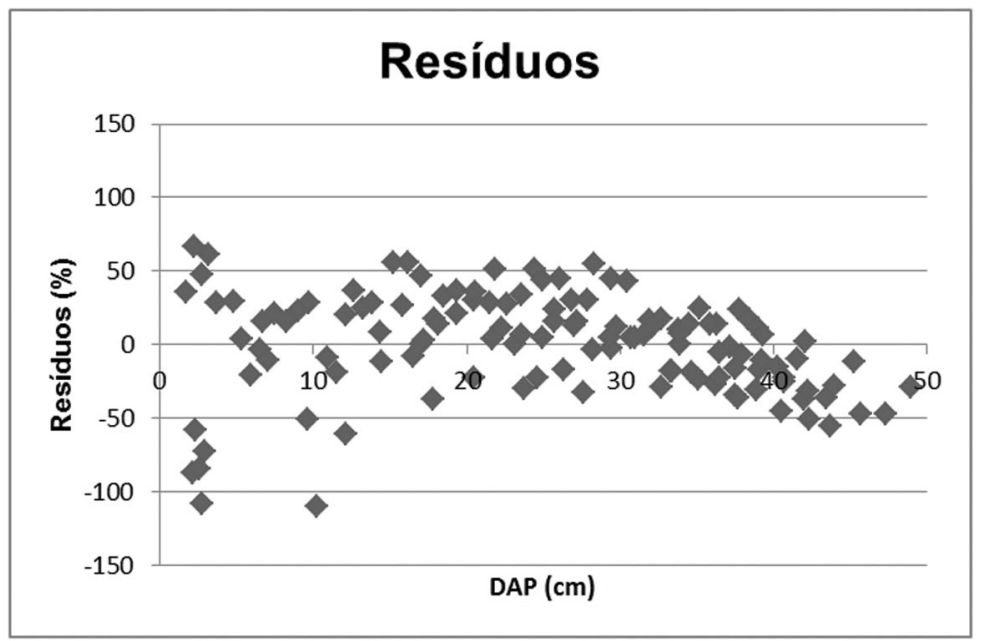

Figura 6. Distribuição dos resíduos para a equação número 1 na estimativa da biomassa das acículas de Pinus elliottii Engelm. Figure 6. Distribution of waste to the equation number 1 in the estimated needles biomass of Pinus elliottii Engelm. 


\subsection{Ajuste de Equações para Estimativa de Biomassa dos Galhos Vivos}

A Tabela 6 apresenta a equação ajustada para estimar a biomassa dos galhos vivos.

A equação logarítmica 1 , que relaciona a biomassa dos galhos vivos de Pinus elliottii Engelm. em função do diâmetro à altura do peito, demonstrou o melhor desempenho, obtido para o coeficiente de determinação $(0,9422)$, erro-padrão da estimativa $(35,5993 \%)$ e o valor de 2021,367 para a estatística F. A distribuição gráfica dos resíduos (Figura 7) evidencia melhor distribuição para o ajuste resultante desse modelo.

Tabela 6. Resultado da equação com melhor estatística para estimativa de biomassa dos galhos vivos em povoamentos de Pinus elliottii Engelm., Clevelândia, PR.

Table 6. Result of the equation with the best statistics to estimate live branches biomass in Pinus elliottii Engelm., Clevelândia, PR.

\begin{tabular}{llllll}
\hline & N. & EQUAÇÃO & $\mathrm{R}^{2}$ aj. & Syx\% & F \\
\hline Biomassa dos galhos vivos & 1 & $\mathrm{y}=0,05414 \mathrm{~d}^{1,78719}$ & 0,9422 & 35,5993 & 2021,36734 \\
\hline
\end{tabular}

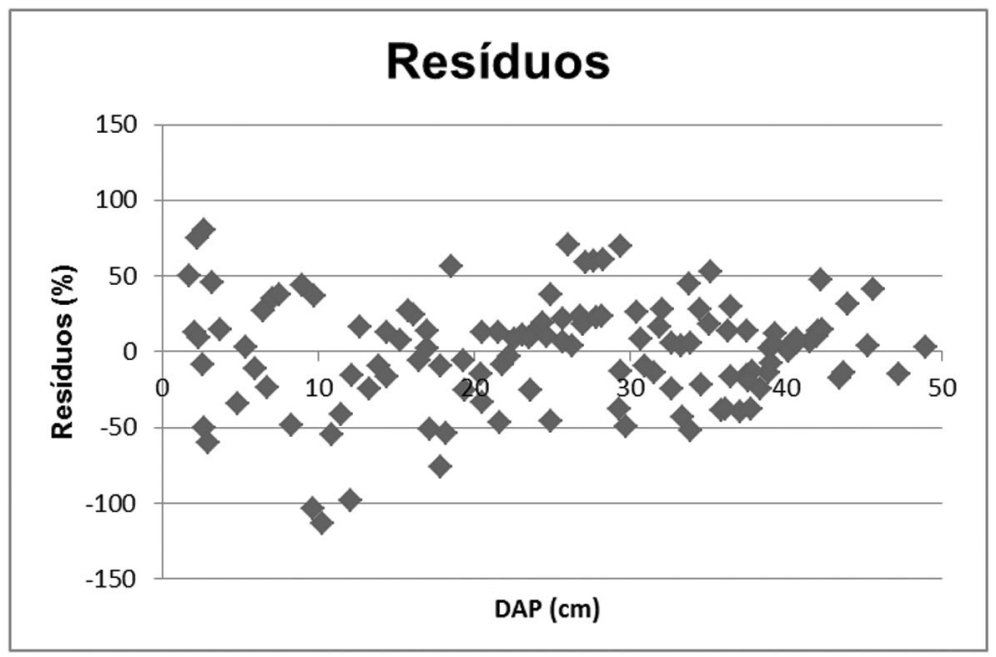

Figura 7. Distribuição dos resíduos para a equação número 1 na estimativa da biomassa dos galhos vivos de Pinus elliottii Engelm. Figure 7. Distribution of waste to the equation number 1 in the estimated live branches biomass of Pinus elliottii Engelm. 


\subsection{Ajuste de Equações para Estimativa da Biomassa da Casca do Fuste}

Os resultados das equações que exprimem a biomassa da casca da árvore de $P$. elliottii, em kg, em função do DAP e altura total, estão exibidos na Tabela 7.

A equação selecionada para a estimativa de biomassa da casca retrata o $\mathrm{R}^{2} \mathrm{aj}$. de 0,9628 e
Syx \% de 34,36\%, cuja distribuição dos resíduos pode ser observada na Figura 8.

As equações resultantes dos modelos logarítmicos 1 e 12, denotaram melhor distribuição gráfica de resíduos para a biomassa e o carbono. No entanto, a equação 1 deve ser considerada como a mais apropriada do ponto de vista prático, visto ser necessária apenas uma variável independente de fácil obtenção.

Tabela 7. Resultado da equação com melhor estatística para estimativa de biomassa da casca do fuste em povoamentos de Pinus elliottii Engelm., Clevelândia, PR.

Table 7. Result of the equation with the best statistics to estimate bark stem biomass in Pinus elliottii Engelm., Clevelândia, PR.

\begin{tabular}{llllll}
\hline & N. & EQUAÇÃO & R 2 aj. & Syx\% & F \\
\hline Biomassa da casca do fuste & 1 & $y=0,01463 \mathrm{~d}^{2,17381}$ & 0,9628 & 34,3631 & 3209,56036 \\
\hline
\end{tabular}

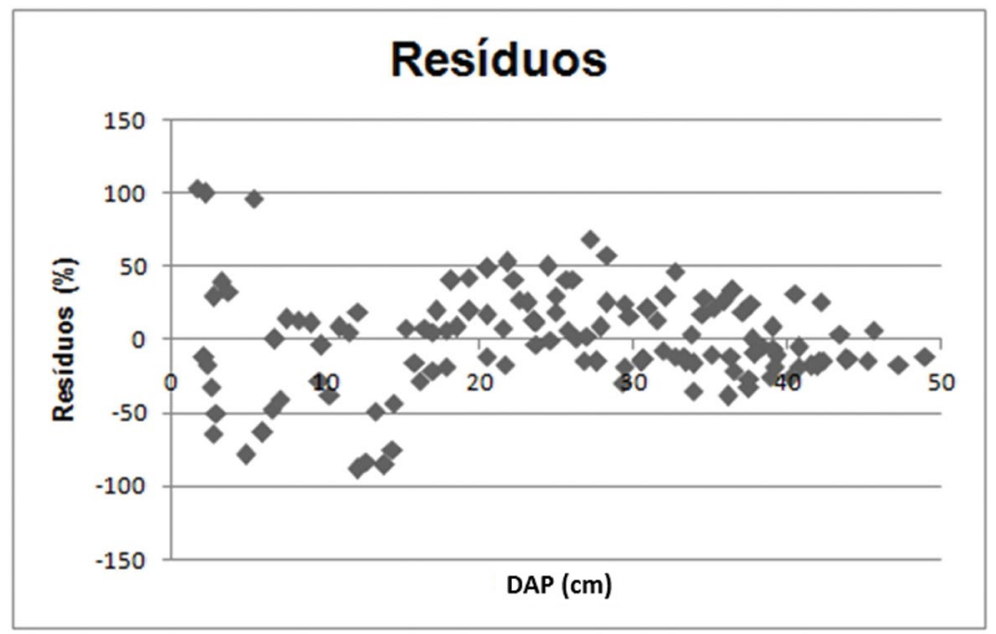

Figura 8. Distribuição dos resíduos para a equação número 1 na estimativa da biomassa da casca do fuste de Pinus elliottii Engelm.

Figure 8. Distribution of waste to the equation number 1 in the estimated bark stem biomass of Pinus elliottii Engelm. 
LISBOA, G. dos S. et al. Quantificação da biomassa Pinus elliottii Engelm.

\subsection{Ajuste de Equações para Estimativa de Biomassa de Raízes}

Os resultados das equações que mostram a biomassa alocada na raiz das árvores de P. elliottii são evidenciados na Tabela 8 .

A equação que apresentou melhor desempenho foi a equação logarítmica 1 , obtendo para biomassa e carbono o coeficiente de determinação 0,9413 e para o erro-padrão da estimativa $31,928 \%$.

A distribuição gráfica dos resíduos (Figura 9) evidencia-se como homogênea para a equação resultante desse modelo.

Tabela 8. Resultado da equação com melhor estatística para estimativa de biomassa de raízes em povoamentos de Pinus elliottii Engelm., Clevelândia, PR.

Table 8. Result of the equation with the best statistics to estimate root biomass in Pinus elliottii Engelm., Clevelândia, PR.

\begin{tabular}{llllll}
\hline & N. & EQUAÇÃO & $\mathrm{R}^{2}$ aj. & Syx\% & F \\
\hline Biomassa das raízes & 1 & $\mathrm{y}=0,13122 \mathrm{~d}^{1,59798}$ & 0,9413 & 31,9282 & 386,1214 \\
\hline
\end{tabular}

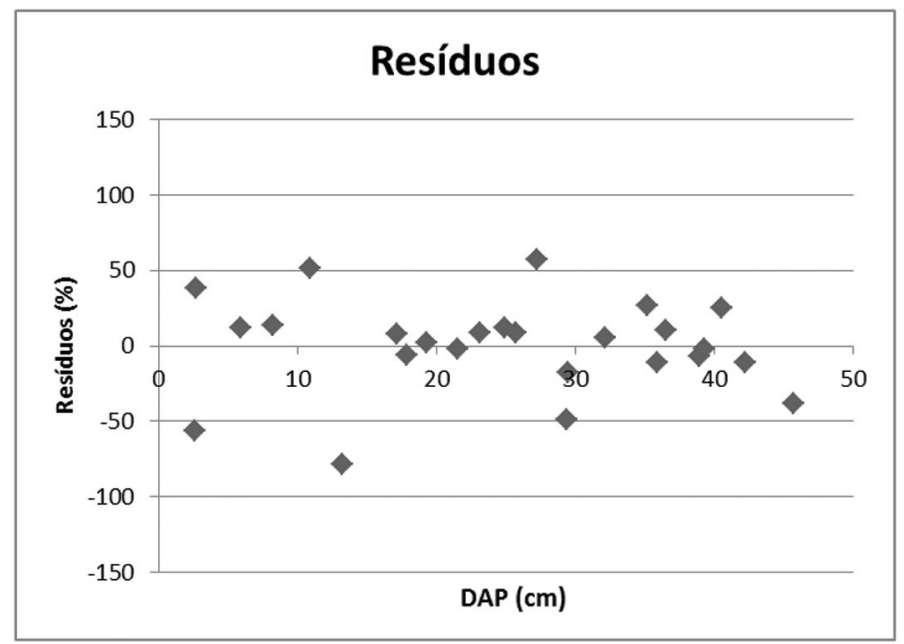

Figura 9. Distribuição dos resíduos para a equação de melhor desempenho na estimativa da biomassa da raiz de Pinus elliottii Engelm.

Figure 9. Distribution of waste for the best performance equation in the estimated root biomass of Pinus elliottii Engelm. 


\section{CONCLUSÕES}

Foi possível gerar estimativas com alta precisão para povoamentos de 2 a 25 anos com a mesma equação, simplificando os procedimentos práticos de inventário de biomassa.

O diâmetro à altura do peito - DAP foi a variável independente mais adequada para a estimativa dos componentes analisados neste estudo.

Entre os modelos testados para a obtenção das estimativas de biomassa e peso de carbono, os ajustes mais adequados foram obtidos com o modelo $\mathrm{y}=\mathrm{b}_{o} \mathrm{~d}^{b}$, que, em geral, apresentou estatística dentro de limites aceitáveis. Todavia, recomenda-se sua aplicação somente dentro da amplitude de diâmetros e alturas incluídas nesta pesquisa, devendo ser considerada a variação abrangida pelos dados amostrais, sendo que seu uso para outras situações e localidades com diferentes sítios implica em teste prévio de exatidão e acuidade dos mesmos.

A análise gráfica dos resíduos permite observar que a utilização de modelos logarítmicos mostrou ser superior aos modelos lineares testados para o peso da biomassa nos distintos componentes.

Os estudos sobre a quantificação de biomassa visando à avaliação do potencial de fixação de carbono por plantações não devem desprezar a determinação do sistema radicular da árvore, visto que sua contribuição para o total é expressiva.

As equações geradas para estimativa de peso total e dos componentes arbóreos da biomassa das árvores de Pinus elliottii Engelm. são importantes ferramentas para análises técnicas, planejamento de projetos e estudos de viabilidade visando participar do mercado de créditos de carbono.

\section{REFERÊNCIAS BIBLIOGRÁFICAS}

BROWN, S.L.; SCHOEDER, P.; KERN, J.S. Spatial distribution of biomass in forests of the eastern USA. Forest Ecology and Management, v. 123, n. 1-3, p. 81-90, 1999.

FAN, S. et al. A large terrestrial carbon sink in North America implied by atmospheric and oceanic carbon dioxide models. Science, n. 282, p. 386-387, 1998.
INSTITUTO BRASILEIRO DE GEOGRAFIA E ESTATÍSTICA - IBGE. Manual técnico da vegetação brasileira. 2. ed. Rio de Janeiro, 2012. 274 p. (Série Manuais Técnicos em Geociências, n. 2).

KETTERINGS, Q.M. et al. Reducing uncertainty in the use of allometric biomass equations for predicting abovegroup tree biomass in mixed secondary forests. Forest Ecology and Management, v. 146, p. 199-209, 2001.

SEDJO, R. A. Temperature forest ecosystem in the global carbon cycle. Ambio, v. 21, p. 274-277, 1992.

WALKER, W. et al. Guia de campo para estimativa de biomassa florestal e estoque de carbono. Versão 1.0. Falmouth: Centro de Pesquisas Woods Hole, 2011. 72 p. 